Supporting information

\title{
Dually Reactive Long Recombinant Linkers for Bioconjugations as an Alternative to PEG
}

Corresponding author Thomas Hoeg-Jensen

Thomas Kjeldsen, Wouter F.J. Hogendorf, Christian W. Tornøe, Jonathan Anderson, Frantisek Hubalek, Carsten E. Stidsen, Jan L. Sorensen, Thomas Hoeg-Jensen*

Novo Nordisk A/S, Novo Nordisk Park H5.S.51, DK-2760 Måløv, Denmark

email tshj@novonordisk.com 
Content:

HPLC and LCMS of (GQAP)49-GQEP-Cys(MPAA) 2

HPLC and LCMS of azido-(GQAP)49-GQEP-Cys(MPAA) 3

HPLC and LCMS of azido-(GQAP)49-GQEP-Cys 4

HPLC and LCMS of B29Ne-iodoacetyl desB30 human insulin 5

HPLC and LCMS of azido-(GQAP)49-GQEP-Cys(B29N $\varepsilon$-acetyl desB30 human insulin) 6

HPLC and LCMS of 14-proparglycine-SFTI peptide 7

HPLC and LCMS of 27-proparglycine-BBI protein $\mathbf{8}$

HPLC and LCMS of SFTI-triazolyl-(GQAP)49-GQEP-Cys(B29N $\varepsilon$-acetyl desB30 human insulin) 9

HPLC and LCMS of BBI-triazolyl-(GQAP)49-GQEP-Cys(B29Ne-acetyl desB30 human insulin) 10
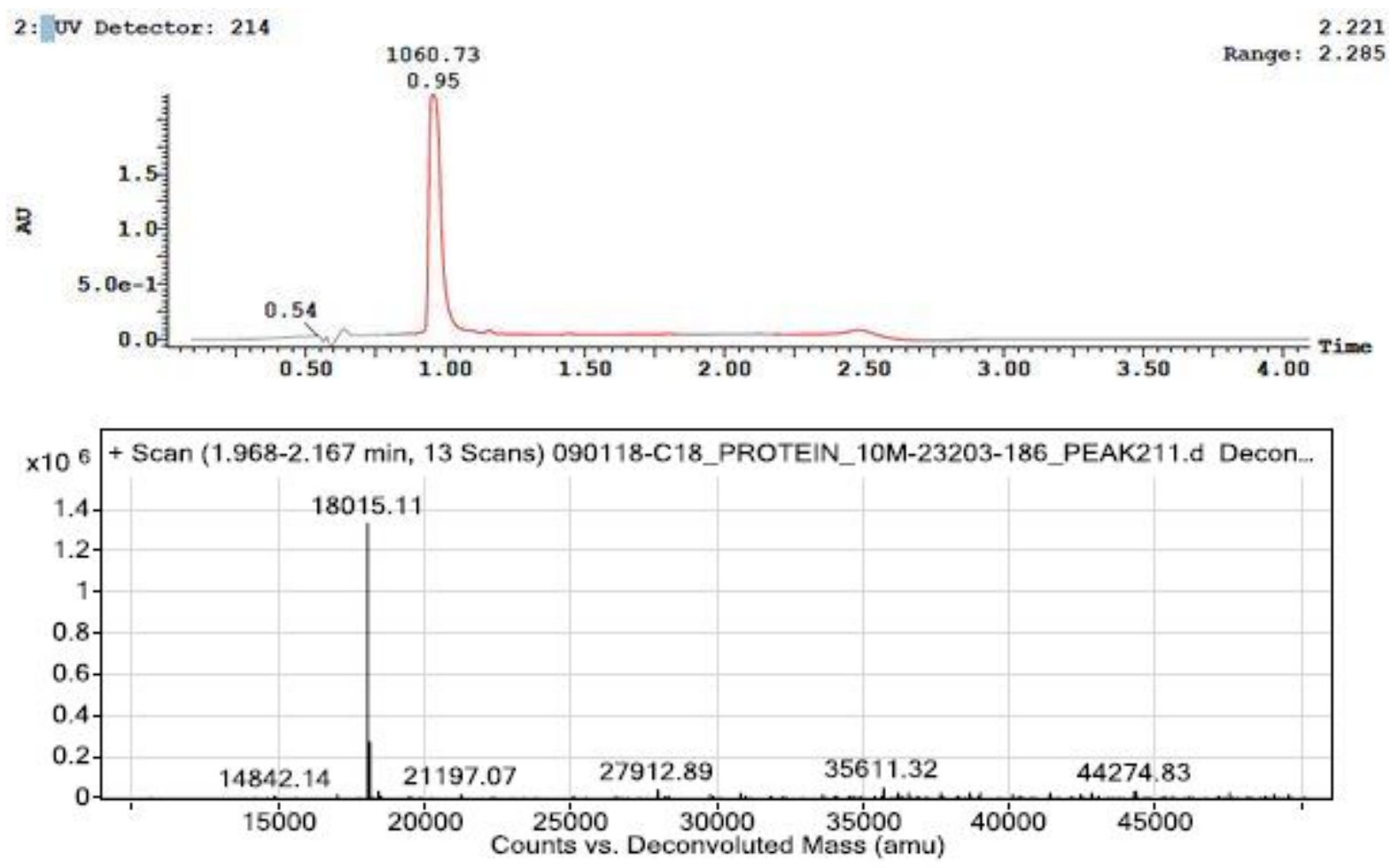

HPLC and LCMS of (GQAP)49-GQEP-Cys(MPAA) 2 

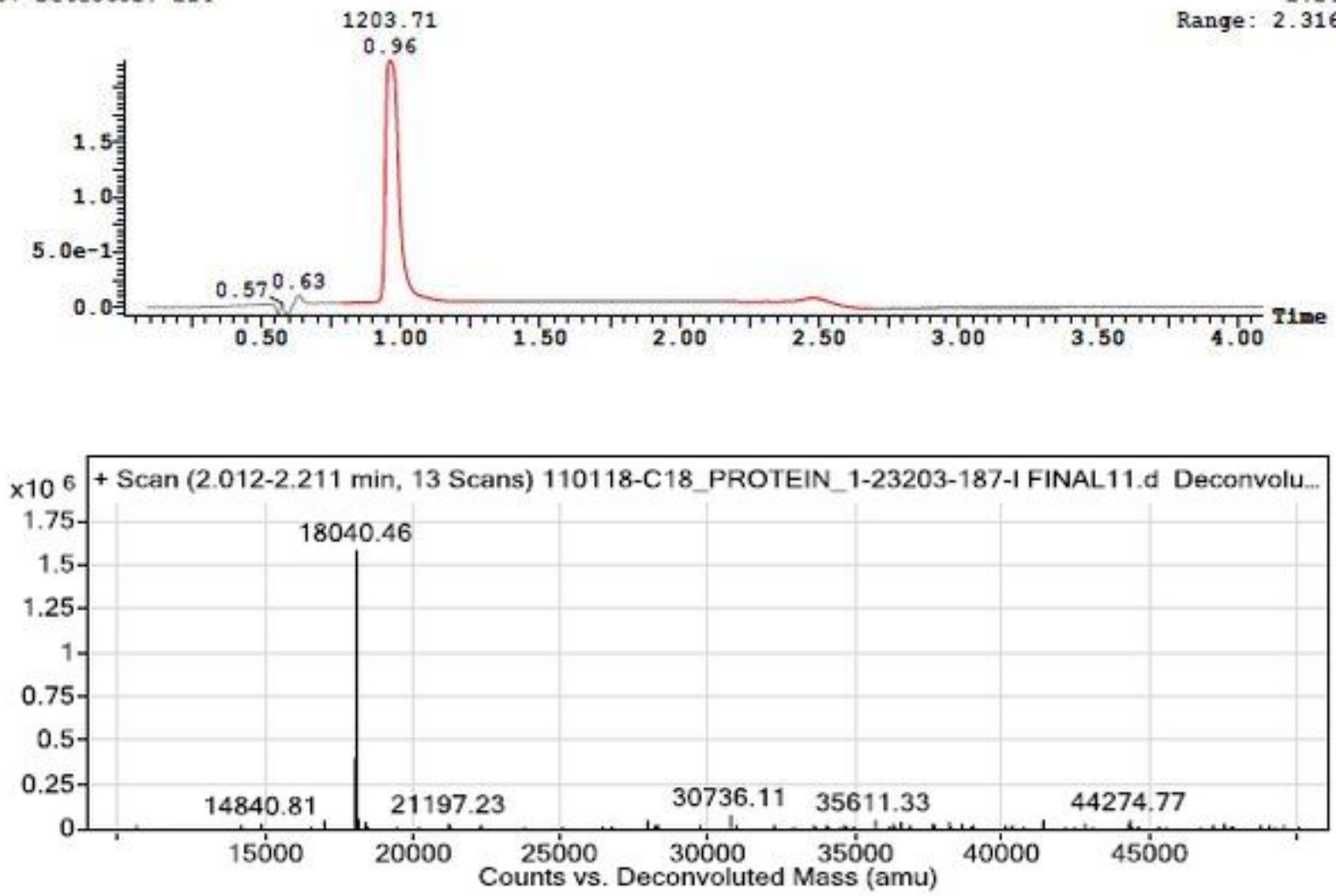

HPLC and LCMS of azido-(GQAP)49-GQEP-Cys(MPAA) 3

uv Detector: 214

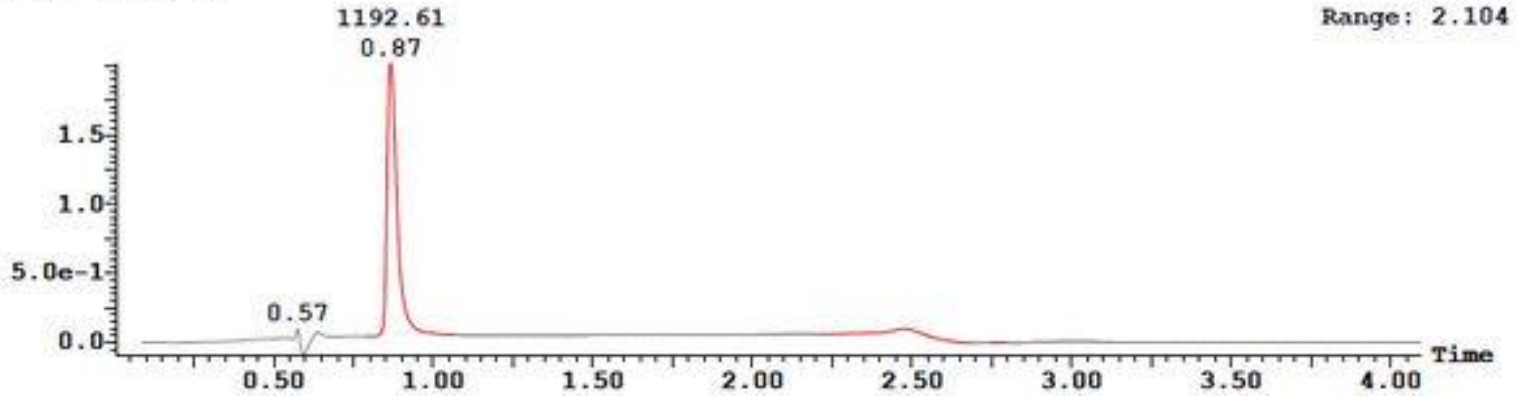




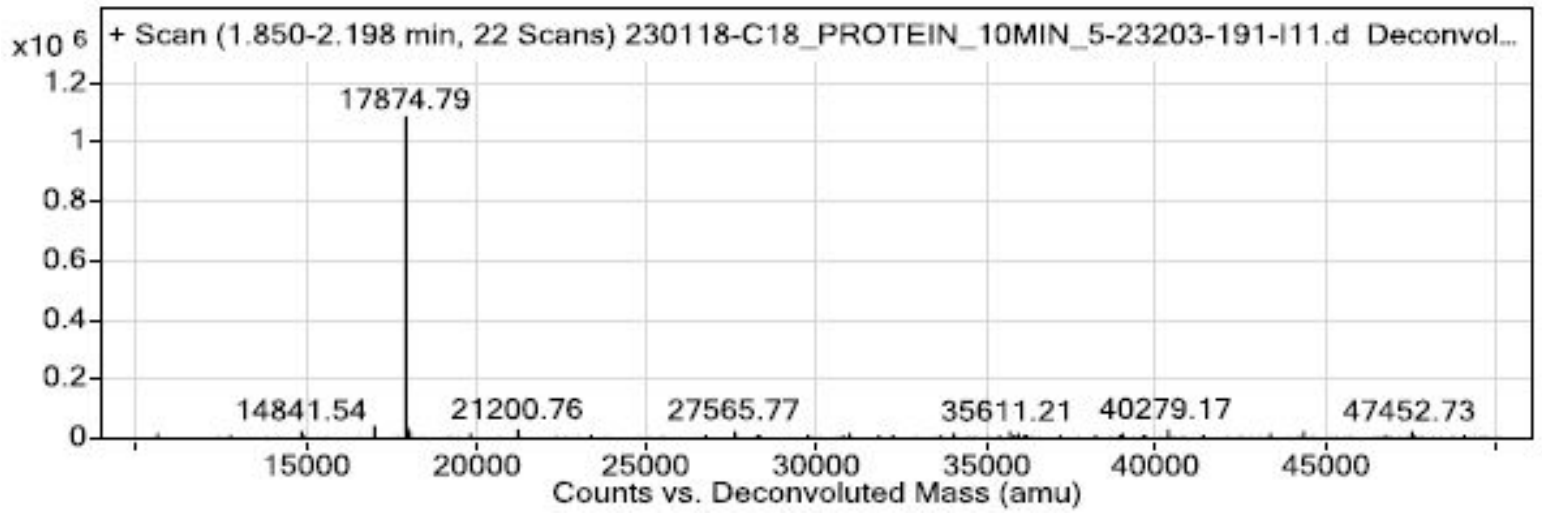

HPLC and LCMS of azido-(GQAP)49-GQEP-Cys 4

2: UV Detector: 214

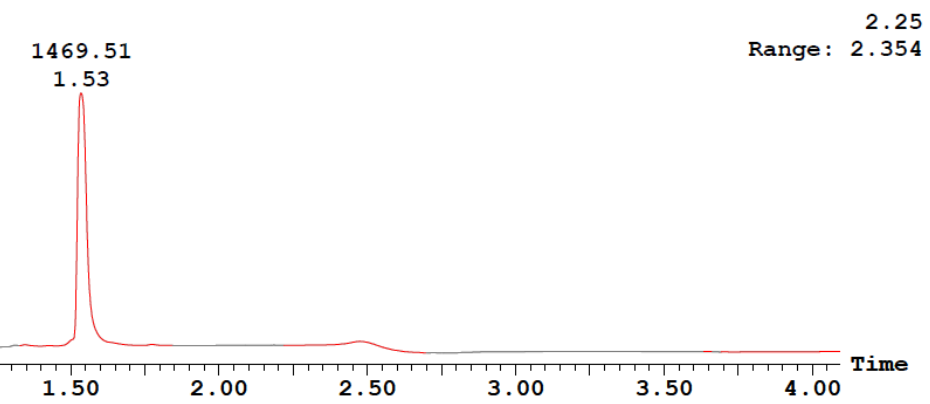

\section{Peak ID Compound Time Mass Found \\ 13 \\ 1.43}

$1:$ MS ES+

$5.3 e+006$

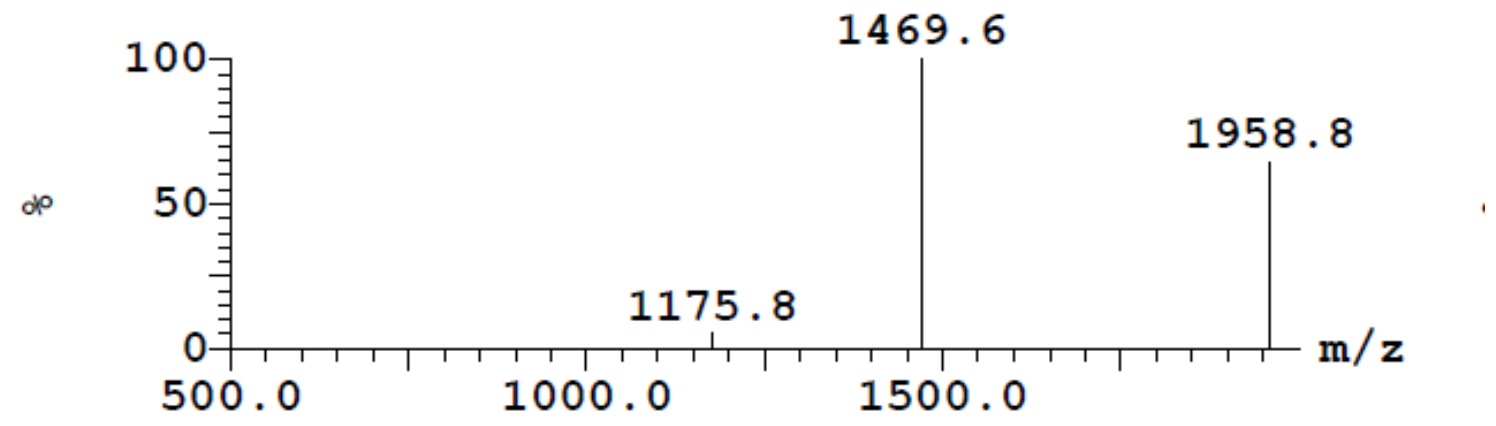

HPLC and LCMS of B29Ne-iodoacetyl desB30 human insulin $\mathbf{5}$ 
UV Detector: 21
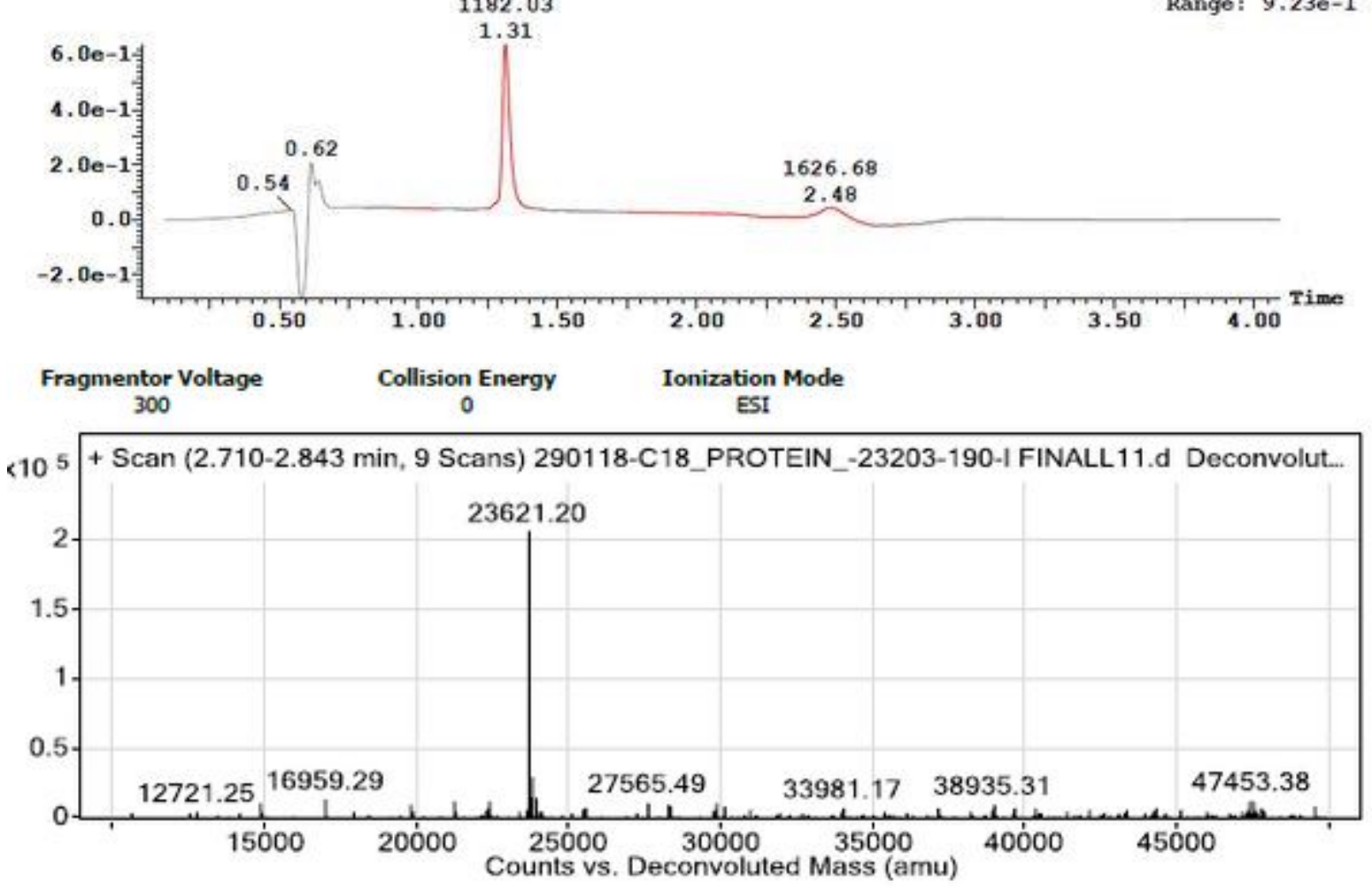

HPLC and LCMS of azido-(GQAP)49-GQEP-Cys(B29Ne-acetyl desB30 human insulin) 6

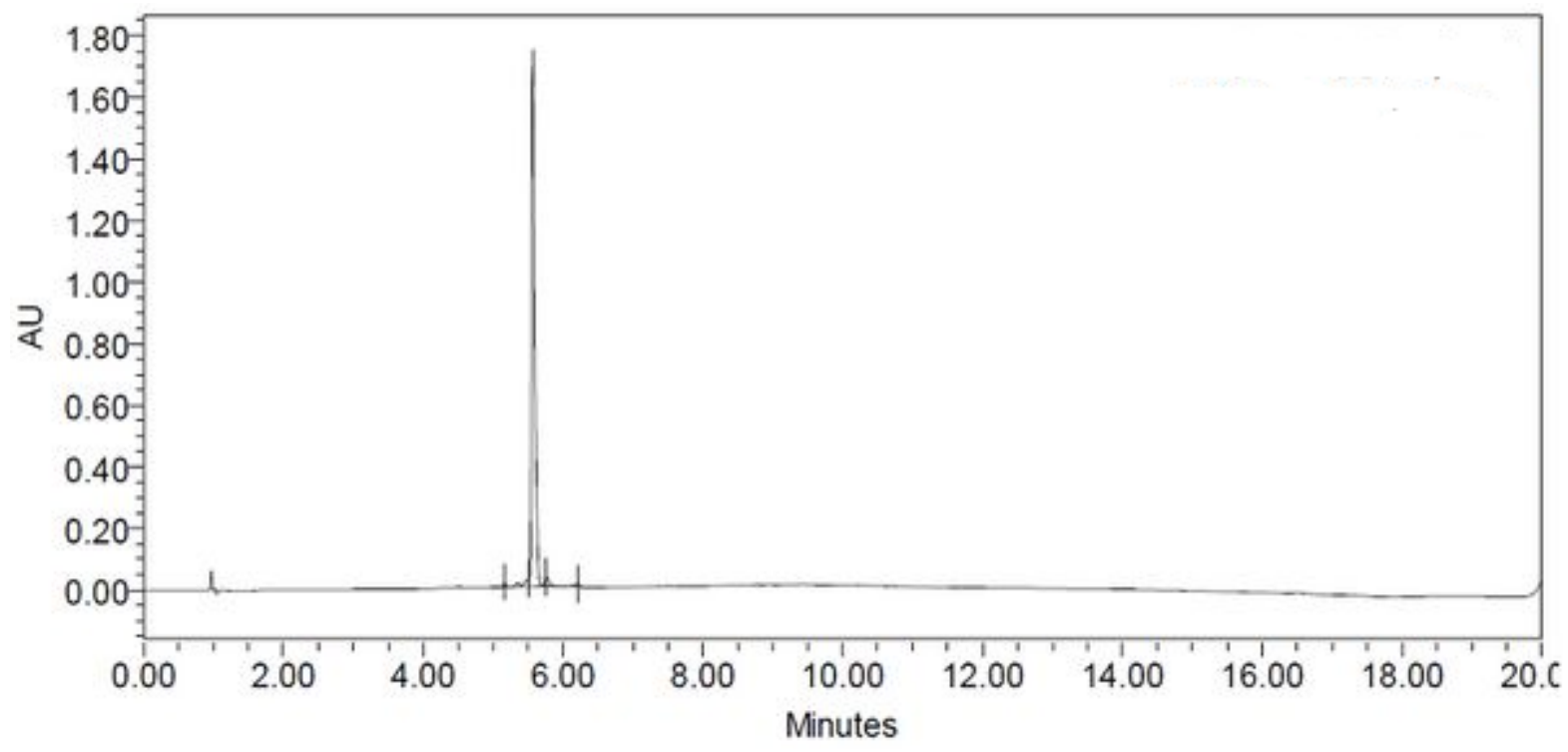




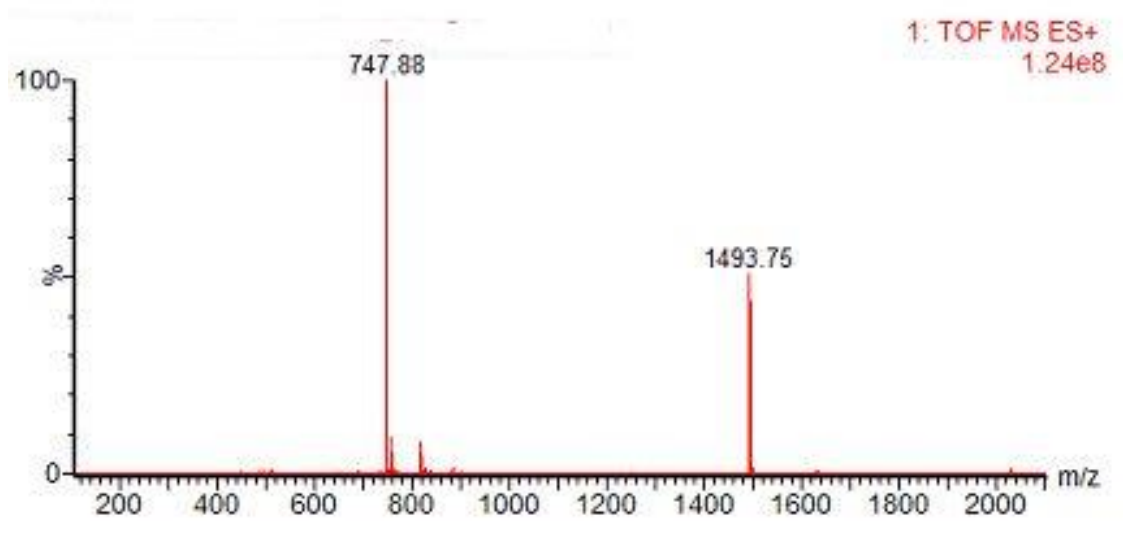

HPLC and LCMS of 14-proparglycine-SFTI peptide 7
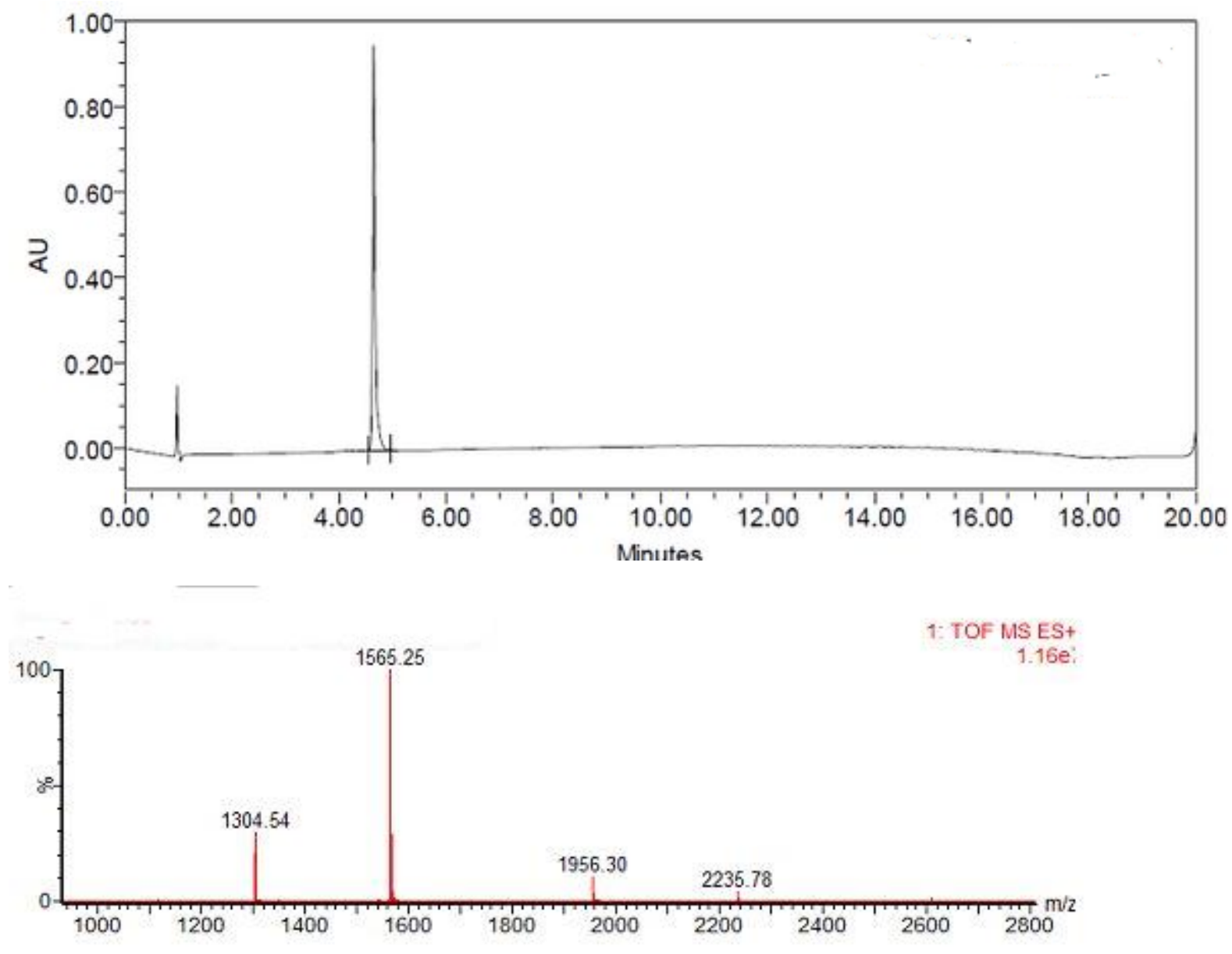

HPLC and LCMS of 27-proparglycine-BBI protein 8 
UV Detector: 214

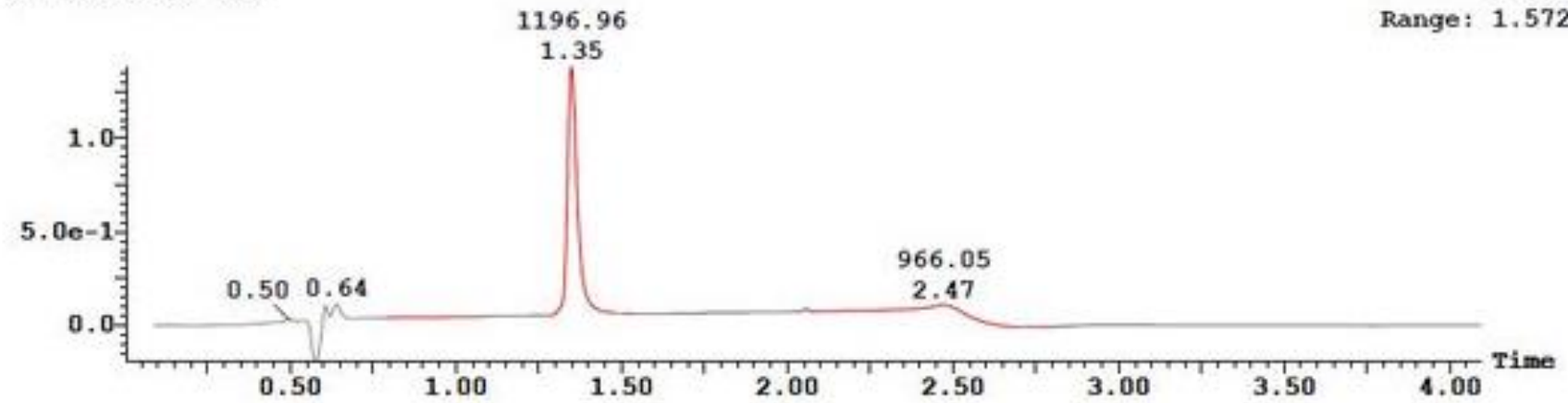

$25114,5 / 21+1=1196,93$

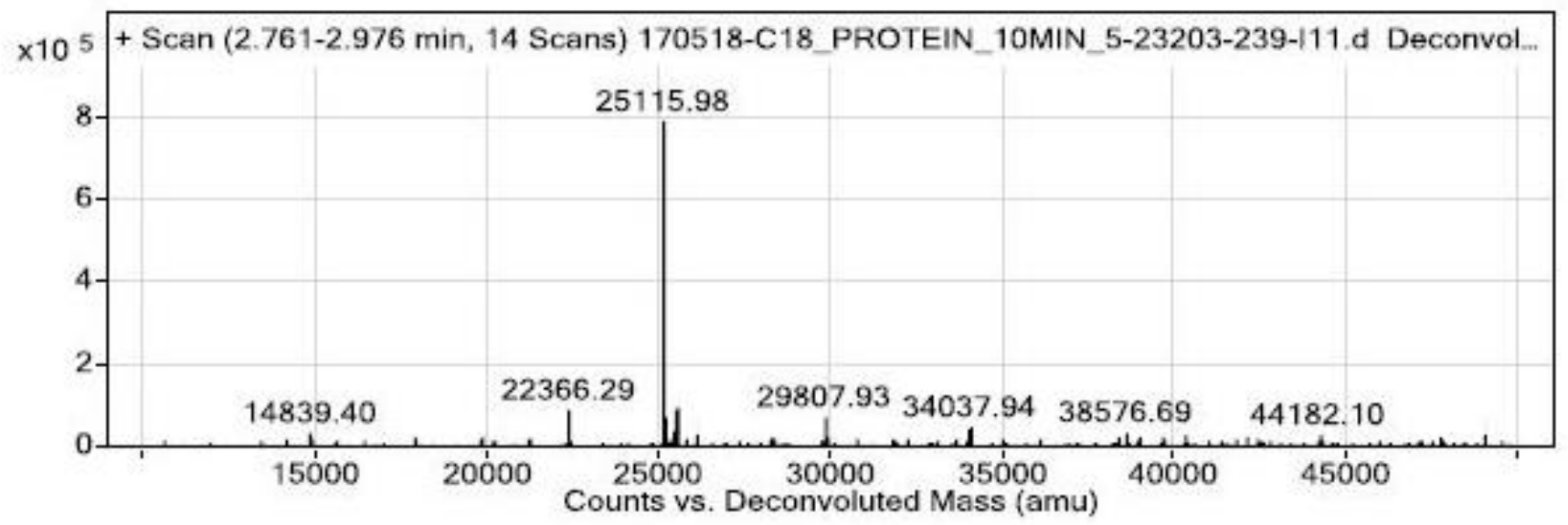

HPLC and LCMS of SFTI-triazolyl-(GQAP)49-GQEP-Cys(B29Ne-acetyl desB30 human insulin) 9 
2: UV Detector: 214

1210.54

Range: 2.228
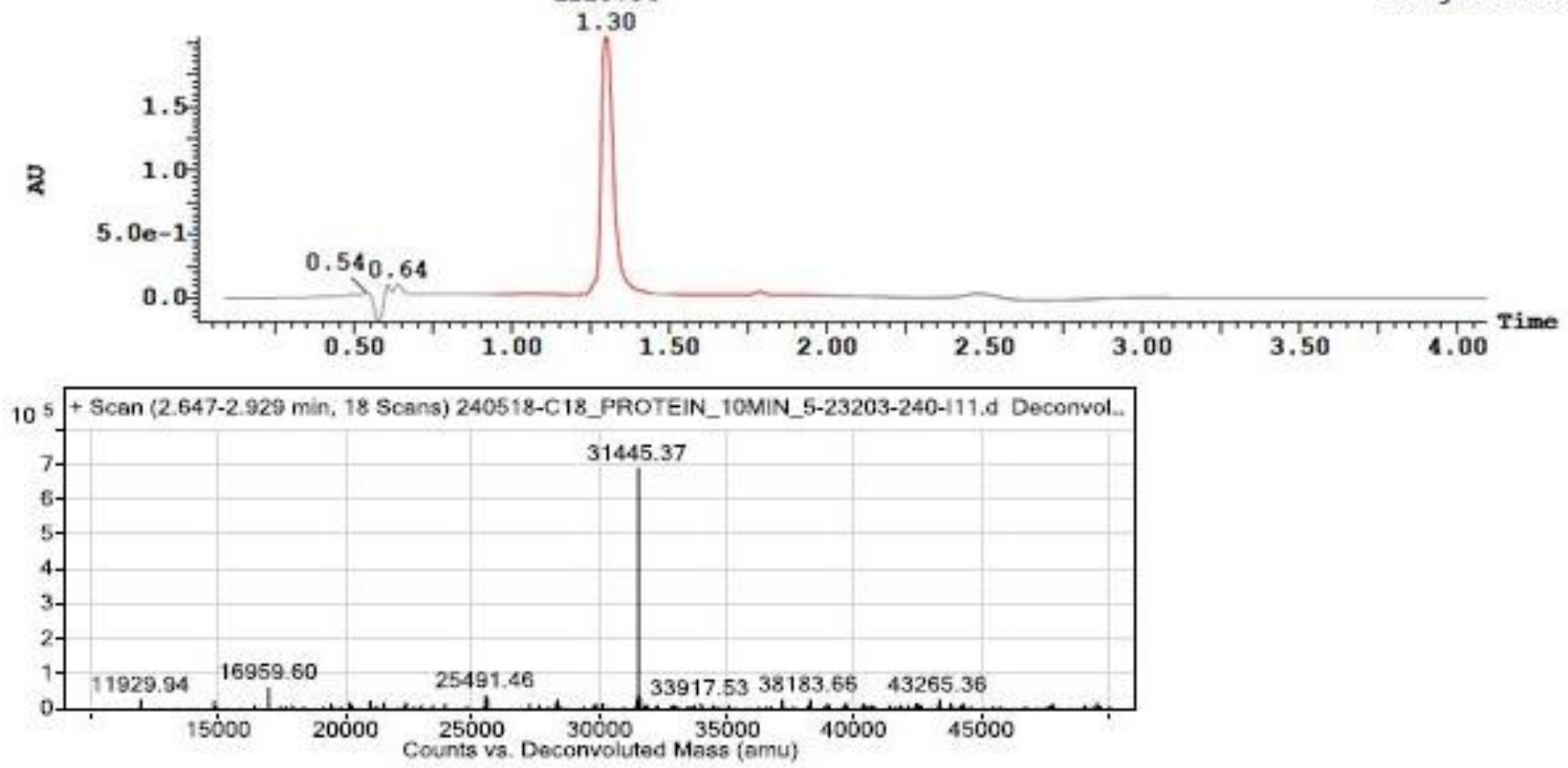

HPLC and LCMS of BBI-triazolyl-(GQAP)49-GQEP-Cys(B29Ne-acetyl desB30 human insulin) 10 